\title{
Soliton solutions of nonlinear fractional differential equations with their applications in mathematical physics
}

\author{
A. C. Cevikel* and E. Aksoy ${ }^{\dagger}$ \\ Yildiz Technical University, Faculty of Arts and Sciences, \\ Department of Mathematics, Istanbul-Turkey. \\ *Tel.: +90 212 3834372; e-mail: acevikel@yildiz.edu.tr †eesinaksoy@gmail.com.tr
}

Received 14 December 2020; accepted 8 January 2021

\begin{abstract}
In this study, the generalized Kudryashov method has been used to investigate a certain type of nonlinear fractional differential equations. Firstly, we proposed a fractional complex transform to convert fractional differential equations into ordinary differential equations. Three applications were given to demonstrate the effectiveness of the present technique. The results show that this method is very effective and powerful mathematical tool for solving nonlinear fractional equations arising in mathematical physics. As a result, abundant types of exact solutions are obtained.
\end{abstract}

Keywords: Exact solutions; modified Riemann-Liouville derivative; fractional complex transform; fractional differential equations.

AMS (2010): 34A08; 83C15.

DOI: https://doi.org/10.31349/RevMexFis.67.422

\section{Introduction}

Nonlinear differential equations appear in many fields, such as in fluid mechanics, viscoelasticity, chemistry, physics, engineering, biology, fluid flow, signal processing, control theory, systems identification, fractional dynamics and finance. Nonlinear phenomena play a crucial role in applied mathematics and physics. It was stated in literature that the nonlinear wave phenomena of dissipation, diffusion, dispersion, reaction and convection have important roles in nonlinear wave equations. New exact solutions might be used to find new phenomena in this field. In the literature, there are many methods to find new exact solutions, such as the tanh-sech method [1], the functional variable method [2-4], the expfunction method $[5,6],\left(G^{\prime} / G\right)$-expansion method $[7,8]$, the generalized exponential rational function method [9-11], the reproducing kernel method [12], Hirota method [13,14] and the ansatz method $[15,16]$.

In recent years, the importance of fractional (nonintegers) differential equations has increased. Fractional calculus is as old as conventional calculus, but is not as popular in science and engineering as conventional calculus. In the last centuries this subject was studied only in mathematics, In recent years it has been used in many fields of engineering and science [17]. Among the investigations for fractional differential equations (FDEs), research for finding approximate/exact solutions of FDEs is an important topic [18-22].

As a result, many methods had been developed such as, the exp-function method [23-26], the $\left(\frac{G^{\prime}}{G}\right)$-expansion method [27-29], the first integral method [30-32], the subequation method [33-36], the functional variable method $[37,38]$ and the simplest equation method [39] so on. With the help of these methods, solutions of FDEs have been established.

Our goal in this work is to present the exact solutions of time-fractional Cahn-Allen equation, space-time fractional Klein-Gordon equation and space-time fractional Zakharov Kuznetsov Benjamin Bona Mahony (ZK-BBM) equation. In Chapter 2, we give preliminaries and notations, in Chapter 3 we describe the algorithm for using the generalized Kudryashov method with fractional complex transform to solve nonlinear FDEs. In Chapter 4, 5 and 6, we obtained exact solutions of time-fractional Cahn-Allen equation [40]

$$
D_{t}^{\alpha} u-u_{x x}+u^{3}-u=0, \quad 0<\alpha \leq 1, \quad t>0
$$

space-time fractional Klein-Gordon equation [35]

$$
\begin{gathered}
D_{t}^{2 \alpha} u+D_{x}^{2 \alpha} u+\gamma u-\beta u^{2}=0, \\
0<\alpha \leq 1, \quad t>0,
\end{gathered}
$$

where $\gamma$ and $\beta$ are a non zero constants. The ZK-BBM equation [35]

$$
\begin{gathered}
D_{t}^{\alpha} u+D_{x}^{\alpha} u-2 a u D_{x}^{\alpha} u-b D_{t}^{\alpha}\left(D_{x}^{2 \alpha} u\right)=0, \\
0<\alpha \leq 1, \quad t>0
\end{gathered}
$$

where $a$ and $b$ are arbitrary constants and $\alpha$ is a parameter describing the order of the fractional derivative.

\section{Preliminaries and Notations}

We give some basic concepts that we will use in this paper. Jumarie's modified Riemann-Liouville derivative of order $\alpha$ is defined as [41] : 


$$
D_{t}^{\alpha} f(t)=\left\{\begin{array}{cc}
\frac{1}{\Gamma(1-\alpha)} \int_{0}^{t}(t-\xi)^{-\alpha-1}(f(\xi)-f(0)) d \xi, & \alpha<0 \\
\frac{1}{\Gamma(1-\alpha)} \frac{d}{d t} \int_{0}^{t}(t-\xi)^{-\alpha}(f(\xi)-f(0)) d \xi, & 0<\alpha<1 \\
\left(f^{(n)}(t)\right)^{(\alpha-n)}, \quad n \leq \alpha<n+1, & n \geq 1 .
\end{array}\right.
$$

Some important properties of the fractional modified Riemann-Liouville derivative were summarized in [42,43]:

$$
D_{t}^{\alpha} t^{r}=\frac{\Gamma(1+r)}{\Gamma(1+r-\alpha)} t^{r-\alpha}, r>0
$$

Similar to integer-order differentiation, the Jumarie's modified fractional differentiation is a linear operation:

$$
D_{t}^{\alpha}(a f(t)+b g(t))=a D_{t}^{\alpha} f(t)+b D_{t}^{\alpha} g(t),
$$

where $a$ and $b$ are constants. The last properties are

$$
\begin{aligned}
D_{t}^{\alpha} c & =0, \quad c=\text { constant } \\
d^{\alpha} x(t) & =\Gamma(1+\alpha) d x(t)
\end{aligned}
$$

We will use these facts in some problems.

\section{The generalized Kudryashov method and the fractional complex transform}

For given general nonlinear FDEs for an unknown function $u$ of independent variables, $x, t$ :

$$
\begin{aligned}
& P\left(u, D_{t}^{\alpha} u, D_{x}^{\beta} u, D_{t}^{\alpha} D_{t}^{\alpha} u, D_{t}^{\alpha} D_{x}^{\beta} u, D_{x}^{\beta} D_{x}^{\beta} u, \ldots\right)=0, \\
& \quad 0<\alpha, \beta \leq 1
\end{aligned}
$$

where $D_{t}^{\alpha} u$ and $D_{x}^{\beta} u$ are the modified Riemann-Liouville derivatives of $u$ with respect to $t$ and $x . P$ is a polynomial of $u=u(x, t)$ and its partial fractional derivatives, in which the highest order derivatives and the nonlinear terms are involved.

$\mathrm{Li}$ and $\mathrm{He}[44,45]$ developed a fractional complex transform to convert FDEs into ordinary differential equations (ODEs). With the help of this transformation, analytical methods devoted to the calculus can be easily applied to the fractional calculus.

We presented the main steps of the generalized Kudryashov method as follows [43]:

Step 1: Firstly, we investigate the travelling wave solution of Eq. (9) as following form;

$$
u(x, t)=U(\xi), \quad \xi=\frac{k x^{\beta}}{\Gamma(1+\beta)}-\frac{c t^{\alpha}}{\Gamma(1+\alpha)},
$$

where $k$ and $c$ are non zero arbitrary constants.

Substituting (10) with (5) into (9), we can rewrite Eq. (9) in the following nonlinear ODE;

$$
Q\left(U, U^{\prime}, U^{\prime \prime}, U^{\prime \prime \prime}, \ldots . .\right)=0 .
$$

where the prime denotes the derivation with respect to $\xi$. We should integrate Eq. (11) term by term one or more times.

Step 2: The exact solutions of Eq. (11) can be obtained in the following form:

$$
\begin{aligned}
U(\xi) & =\sum_{i=0}^{N} a_{i} F^{i}(\xi)=a_{0}+a_{1} F(\xi) \\
& +a_{2} F^{2}(\xi)+\ldots+a_{N} F^{N}(\xi)
\end{aligned}
$$

where $a_{i}$ are unknown constants, $F(\xi)$ is the function as:

$$
F(\xi)=\frac{1}{1 \pm e^{\xi}}
$$

This function satisfies to first order the ordinary differential equation

$$
F_{\xi}=F^{2}-F
$$

We will use Eq. (14) to calculate the derivatives of the function $U(\xi)$.

Step 3: We should calculate the derivatives of function $U(\xi)$. As an example we consider the general case when $N$ is arbitrary. Taking into consideration Eq. (12), we obtain

$$
\begin{aligned}
U_{\xi} & =\sum_{i=1}^{N} a_{i} i(F-1) F^{i}, \\
U_{\xi \xi} & =\sum_{i=1}^{N} a_{i} i\left[(i+1) F^{2}-(2 i+1)(F-1)\right] F^{i} .
\end{aligned}
$$

The higher order derivatives of function $U(\xi)$ can be found in [46].

Step 4: We substitute the expression (14) in Eq. (11). Later, we take $U(\xi)$ from Eq. (12) into account. Thus, Eq. (9) takes the form

$$
p[F(\xi)]=0,
$$

where $p[F(\xi)]$ is a polynomial of function $F(\xi)$. We collect all terms with the same powers of function $F(\xi)$ and equate these expressions equal to zero. Thus, we get a system of algebraic equations. By solving this system, we obtain the exact solutions of Eq. (9). 


\section{Applications}

We obtained the exact solutions of three fractional differential equations by using the generalized Kudryashov method.

\subsection{The time-fractional Chan-Allen equation}

We consider the travelling wave solutions of Eq. (1) and we perform the transformation $u(x, t)=u(\xi)$ and

$$
\xi=k x-\frac{c t^{\alpha}}{\Gamma(1+\alpha)},
$$

where $k$ and $c$ are nonzero constants. Plugging Eq. (18) in (1), the equation can be reduced to an ODE,

$$
c u^{\prime}+k^{2} u^{\prime \prime}-u^{3}+u=0,
$$

where $u^{\prime}=d u / d \xi$. Using the Eq. (19), for the linear term of highest order $u^{\prime \prime}$ with the highest order nonlinear term $u^{2}$. We have that, balancing $u^{\prime \prime}$ with $u^{2}$ in Eq. (19) gives

$$
N+2=3 N \Rightarrow N=1 \text {. }
$$

Thus, we have

$$
u(\xi)=a_{0}+a_{1} F(\xi) .
$$

By substituting Eq. (21) into (19) using Eq. (13) and then setting the coefficients of $F^{i}(i=0,1,2,3)$ to be zero, we get algebraic equations about $a_{0}, a_{1}, c$ and $k$. If we solve these algebraic equations:

Case 1:

$$
a_{0}=0, \quad a_{1}=-1, \quad c=\frac{3}{2} \quad k= \pm \frac{1}{2}
$$

Case 2:

$$
a_{0}=0, \quad a_{1}=1, \quad c=\frac{3}{2} \quad k= \pm \frac{1}{2}
$$

Case 3:

$$
a_{0}=1, \quad a_{1}=-1, \quad c=-\frac{3}{2} \quad k= \pm \frac{1}{2}
$$

Case 4:

$$
a_{0}=-1, \quad a_{1}=1, \quad c=-\frac{3}{2} \quad k= \pm \frac{1}{2}
$$

When we substitute Eqs. (22)-(25), we have the following solutions of FDE (1):

$$
\begin{aligned}
& u_{1}(x, t)=-\frac{1}{1+\cosh \left[ \pm \frac{x}{\sqrt{2}}-\frac{3 t^{\alpha}}{2 \Gamma(1+\alpha)}\right]+\sinh \left[ \pm \frac{x}{\sqrt{2}}-\frac{3 t^{\alpha}}{2 \Gamma(1+\alpha)}\right]}, \\
& u_{2}(x, t)=\frac{1}{1+\cosh \left[ \pm \frac{x}{\sqrt{2}}-\frac{3 t^{\alpha}}{2 \Gamma(1+\alpha)}\right]+\sinh \left[ \pm \frac{x}{\sqrt{2}}-\frac{3 t^{\alpha}}{2 \Gamma(1+\alpha)}\right]}, \\
& u_{3}(x, t)=1-\frac{1}{1+\cosh \left[ \pm \frac{x}{\sqrt{2}}+\frac{3 t^{\alpha}}{2 \Gamma(1+\alpha)}\right]+\sinh \left[ \pm \frac{x}{\sqrt{2}}+\frac{3 t^{\alpha}}{2 \Gamma(1+\alpha)}\right]}, \\
& u_{4}(x, t)=-1+\frac{1}{1+\cosh \left[ \pm \frac{x}{\sqrt{2}}+\frac{3 t^{\alpha}}{2 \Gamma(1+\alpha)}\right]+\sinh \left[ \pm \frac{x}{\sqrt{2}}+\frac{3 t^{\alpha}}{2 \Gamma(1+\alpha)}\right]}
\end{aligned}
$$

Remark 1: We get the hyperbolic function solutions of the time-fractional Chan-Allen equation and these obtained exact solutions are soliton solutions.

\subsection{The space-time fractional Klein-Gordon equation}

We will investigate the travelling wave solutions of Eq. (2), for which we implement the transformation $u(x, t)=u(\xi)$ and

$$
\xi=\frac{k x^{\alpha}}{\Gamma(1+\alpha)}-\frac{c t^{\alpha}}{\Gamma(1+\alpha)},
$$

where $k$ and $c$ are nonzero constants. Combining Eqs. (2) and (30) reduces the equation to an ODE,

$$
\left(c^{2}-k^{2}\right) u^{\prime \prime}+\gamma u-\beta u^{2}=0,
$$

where $u^{\prime}=d u / d \xi$. Setting Eqs. (12) and (16) into Eq. (31) and balancing the highest order nonlinear terms of $u^{\prime \prime}$ and $u^{2}$ in Eq. (31),

$$
N+2=2 N \Rightarrow N=2 .
$$

Therefore, the solution of Eq. (32) can be expressed as

$$
u=a_{0}+a_{1} F(\xi)+a_{2} F^{2}(\xi) .
$$

Upon substitution of Eq. (33) into (31), the use of Eq. (13) and setting the coefficients of $F^{i}(i=0,1,2,3,4)$ to zero, we get

\section{Case 1:}

$$
a_{0}=\frac{\gamma}{\beta}, \quad a_{1}=-\frac{6 \gamma}{\beta}, \quad a_{2}=\frac{6 \gamma}{\beta} \quad c= \pm \sqrt{k^{2}+\gamma}
$$

Case 2:

$$
a_{0}=0, \quad a_{1}=\frac{6 \gamma}{\beta}, \quad a_{2}=-\frac{6 \gamma}{\beta} \quad c= \pm \sqrt{k^{2}-\gamma}
$$




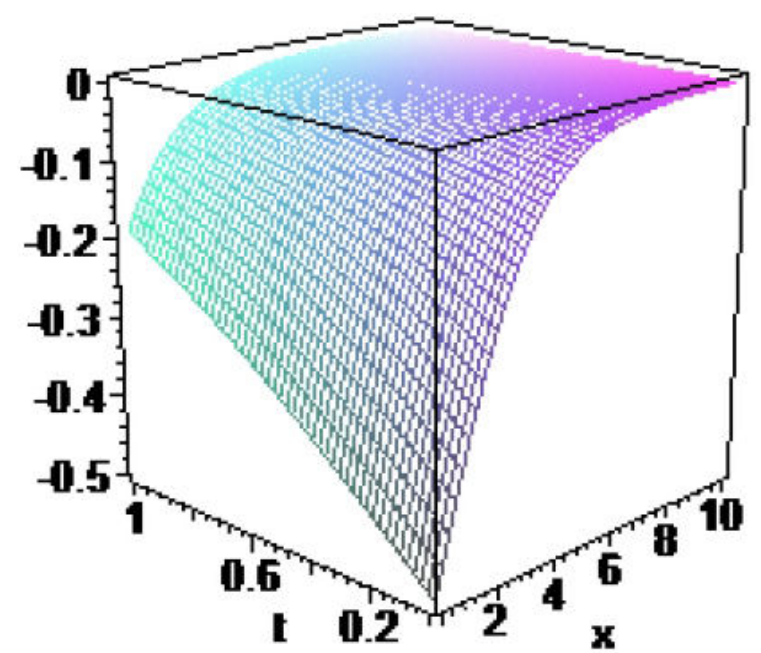

FIGURE 1. Solution of Eq. (1).

Inserting Eqs. (34) and (35) into Eq. (33), we obtain the following exact solutions of Eq. (2):

$$
\begin{aligned}
& u_{1}(x, t)=\frac{\gamma}{\beta}\left\{\frac{\cosh \left[\frac{k x^{\alpha}}{\Gamma(1+\alpha)} \pm \frac{\sqrt{k^{2}+\gamma} t^{\alpha}}{\Gamma(1+\alpha)}\right]-2}{\cosh \left[\frac{k x^{\alpha}}{\Gamma(1+\alpha)} \pm \frac{\sqrt{k^{2}+\gamma} t^{\alpha}}{\Gamma(1+\alpha)}\right]+1}\right\}, \\
& u_{2}(x, t)=\frac{3 \gamma}{\beta\left\{1+\cosh \left[\frac{k x^{\alpha}}{\Gamma(1+\alpha)} \pm \frac{\sqrt{k^{2}-\gamma} t^{\alpha}}{\Gamma(1+\alpha)}\right]\right\}} .
\end{aligned}
$$

Remark 2: We have the hyperbolic function solutions of the space-time fractional Klein-Gordon equation. These obtained exact solutions are soliton solutions and periodic solitary wave solutions.

\subsection{The space-time fractional ZK-BBM equation}

We want to find traveling wave solutions of the Eq. (3), reason for which we apply the transformation $u(x, t)=u(\xi)$ and

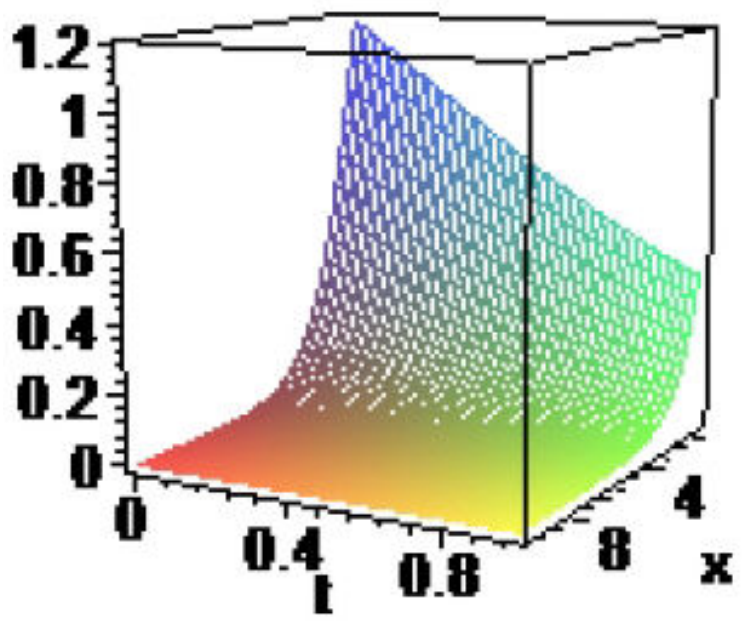

FIGURE 2. Solution Eq. (2) when $\gamma=1, \beta=1, k=1$. $\xi=\left(k x^{\alpha} / \Gamma(1+\alpha)\right)-\left(c t^{\alpha} / \Gamma(1+\alpha)\right)$, where $k$ and $c$ are nonzero constants. Then, by integrating this equation once with respect to $\xi$ and setting the integration constant to zero, we attain,

$$
(k-c) u-a k u^{2}+b c k^{2} u^{\prime \prime}=0,
$$

where $u^{\prime}=d u / d \xi$. Balancing $u^{\prime \prime}$ with $u^{3}$ gives $N=$ 1.Therefore, the solution of Eq. (19) can be expressed as

$$
u=a_{0}+a_{1} F(\xi)+a_{2} F^{2}(\xi) .
$$

Substituting Eqs. (13) and (39) into Eq. (38), collecting all coefficients for each power of $F^{i}(i=0,1,2,3,4)$, and setting each of the coefficients to zero, we get a system of algebraic equations with the following solutions:

Case 1:

$$
\begin{aligned}
& a_{0}=0, \quad a_{1}=\frac{6 b k^{2}}{a\left(b k^{2}-1\right)} \\
& a_{2}=-\frac{6 b k^{2}}{a\left(b k^{2}-1\right)} \quad c=-\frac{k}{b k^{2}-1} .
\end{aligned}
$$

Case 2:

$$
\begin{aligned}
& a_{0}=\frac{b k^{2}}{a\left(1+b k^{2}\right)}, \quad a_{1}=-\frac{6 b k^{2}}{a\left(1+b k^{2}\right)}, \\
& a_{2}=\frac{6 b k^{2}}{a\left(1+b k^{2}\right)} \quad c=\frac{k}{1+b k^{2}} .
\end{aligned}
$$

Inserting Eqs. (40) and (41) into Eq. (39), we obtain the following exact solutions of Eq. (3):

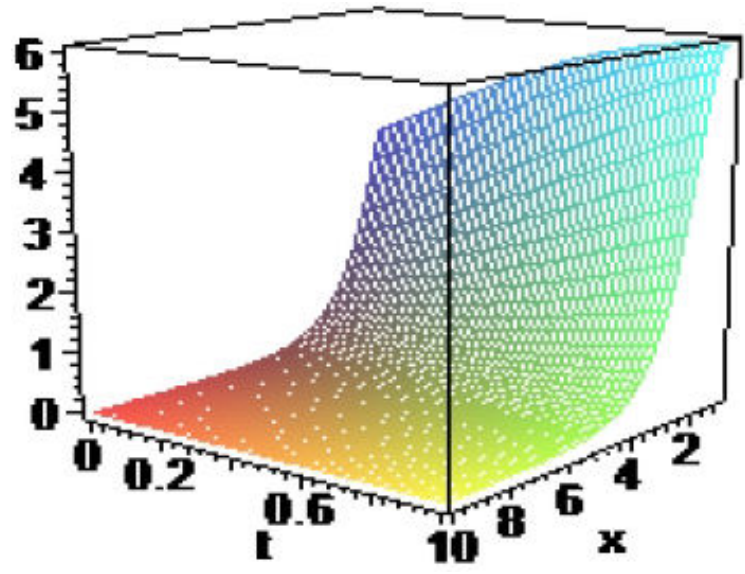

FIGURE 3. Solution of Eq. (3) when $b=2, k=1, a=1, c=-1$. 


$$
\begin{aligned}
u_{1}(x, t) & =\frac{3 b k^{2}}{a\left(b k^{2}-1\right)} \\
& \times\left\{\frac{1}{\cosh k\left[\frac{x^{\alpha}}{\Gamma(1+\alpha)}+\frac{c t^{\alpha}}{\left(b k^{2}-1\right) \Gamma(1+\alpha)}\right]}\right\}, \\
u_{2}(x, t) & =\frac{b k^{2}}{a\left(1+b k^{2}\right)} \\
\times & \left\{\frac{\cosh k\left[\frac{x^{\alpha}}{\Gamma(1+\alpha)}+\frac{c t^{\alpha}}{\left(1+b k^{2}\right) \Gamma(1+\alpha)}\right]-2}{\cosh k\left[\frac{x^{\alpha}}{\Gamma(1+\alpha)}+\frac{c t^{\alpha}}{\left(1+b k^{2}\right) \Gamma(1+\alpha)}\right]+1}\right\} .
\end{aligned}
$$

Remark 3: We obtain the hyperbolic function solutions of the space-time fractional ZK-BBM equation. In particular, the soliton solutions, compacton solutions, peakon solutions and other solutions have been found for such physical problems.

\section{Conclusion}

In this paper, the generalized Kudryashov method has been successfully applied to find the solution of time-fractional Cahn-Allen equation, space-time fractional Klein-Gordon equation and space-time fractional ZK-BBM equation. Calculations in generalized Kudryashov method are simple, reliable and effective mathematical tool for solving fractional differential equations in science and engineering. As far as we know, the solutions obtained are new solutions that are not available in the literature. The solutions obtained will play a significant role in explaining many physical problems. According to the results obtained, this approach can be used to other nonlinear FDEs, nonlinear fractional equation systems, the fractional complex equations, the fractional difference equations, etc. All the solutions reported above have been verified using the symbolic computation system Maple.
1. A. Bekir and A. C. Cevikel, Solitary wave solutions of two nonlinear physical models by tanh-coth method, Commun. Nonlinear Sci. Numer. Simul., 14, (2009) 1804, https://doi. org/10.1016/j.cnsns.2008.07.004.

2. M. Inc et al., New solitary wave solutions for the conformable Klein-Gordon equation with quantic nonlinearity. AIMS Math., 5 (2020) 6972. https://doi.org/10. $3934 /$ math.2020447.

3. A. C. Cevikel, A. Bekir, S. San, and M. B. Gucen, Construction of periodic and solitary wave solutions for the complex nonlinear evolution equations, J. Franklin Inst., 2 (2014) 694, https://doi.org/10.1016/j. jfranklin.2013.04.017.

4. A. C. Cevikel, A. Bekir, M. Akar, and S. San, A procedure to construct exact solutions of nonlinear evolution equations, Pramana 79 (2012) 3337. https: / / doi.org/10.1007/ s12043-012-0326-1.

5. A. Bekir and A. C. Cevikel, New solitons and periodic solutions for nonlinear physical models in mathematical physics, Nonlinear Anal. Real World Appl. 11 (2010) 3275. https: //doi.org/10.1016/j.nonrwa.2009.10.015.

6. A. C. Cevikel and A. Bekir, New Solitons and Periodic Solutions for $(2+1)$-dimensional Davey-Stewartson Equations, Chin. J. Phys., 51 (2013) 11. https://doi.org/10. 6122 /CJP. 51.1.

7. M. Inc et al., New exact solutions for the Kaup-Kupershmidt equation, AIMS Math. 5 (2020) 6726, https://doi.org/ $10.3934 / \mathrm{math} .2020432$.

8. A. Bekir and A. C. Cevikel, New exact travelling wave solutions of nonlinear physical models, Chaos Solitons Fractals 41 (2009) 1733. https://doi.org/10.1016/j.chaos. 2008.07 .017$.

9. B. Ghanbari, H. GÃ $\frac{1}{4}$ nerhan, and S. Momani, Exact optical solutions for the regularized long-wave Kadomtsev-Petviashvili equation, Phys. Scr., 95 (2020) https://doi.org/10. $1088 / 1402-4896 / \mathrm{abb} 5 \mathrm{c} 8$.

10. B. Ghanbari, M. S. Osman, and D. Baleanu, Generalized exponential rational function method for extended ZakharovKuzetsov equation with conformable derivative, Mod. Phys. Lett. A 34 (2019) 195. https://doi.org/10.1142/ S0217732319501554.

11. B. Ghanbari and C.-K. Kuo, New exact wave solutions of the variable-coefficient $(1+1)$-dimensional BenjaminBona-Mahony and $(2+1)$-dimensional asymmetric Nizhnik-Novikov-Veselov equations via the generalized exponential rational function method, Eur. Phys. J. Plus 134 (2019) 334. https://doi.org/10.1140/epjp/ i2019-12632-0.

12. B. Ghanbari and A. Akgül, Abundant new analytical and approximate solutions to the generalized Schamel equation, Eur. Phys. J. Plus, 95 (2020) 7. https : / / doi.org/10.1088/ 1402-4896/ab8b27.

13. C.-K. Kuo and B. Ghanbari, Resonant multi-soliton solutions to new $(3+1)$-dimensional Jimbo-Miwa equations by applying the linear superposition principle, Nonlinear Dyn. 96 (2019) 459. https://doi.org/10.1007/ s11071-019-04799-9.

14. B. Ghanbari and C.-K. Kuo,, A variety of solitary wave solutions to the $(2+1)$-dimensional bidirectional SK and variablecoefficient SK equations, Res. Phys. 18 (2020) 103. https: //doi.org/10.1016/j.rinp.2020.103266.

15. A. Bekir, A. C. Cevikel, Ö. G'"uner and S. San, Bright and Dark Soliton Solutions of the (2+1)-Dimensional Evolution Equations, Math. Model. Anal., 19 (2014) 118. https:// doi.org/10.3846/13926292.2014.893456.

16. Ö. Güner, A. Bekir, and A. C. Cevikel, Dark soliton and periodic wave solutions of nonlinear evolution equations, Adv. Differ. Equ.,2013 (2013) 68. https://doi.org/10.1186/ 1687-1847-2013-68. 
17. R. Hilfer, Applications of Fractional Calculus in Physics, (World Scientific, River Edge, New Jersey, 2000). pp. 171-203.

18. A. Kilbas, H. M. Srivastava, and J. J. Trujillo, Theory and Applications of Fractional Differential Equations, 1st Edition, (Elsevier, Amsterdam 2006) pp. 1-540.

19. S. Miller and B. Ross, An Introduction to the Fractional Calculus and Fractional Differential Equations, 1st Edition, (Wiley, New York 1993), pp. 1-384.

20. I. Podlubny, Fractional Differential Equations, 1st Edition, (Academic Press, California 1999), pp. 1-340.

21. J. Sabatier, O. P. Agrawal, and J. A. T. Machado, Advances in Fractional Calculus: Theoretical Developments and Applications in Physics and Engineering, 1st Edition, (Springer, Dordrecht, 2007), pp. 27-42

22. R. M. Jena, S. Chakraverty, H. Rezazadeh, and D. D. Ganji, On the solution of time-fractional dynamical model of Brusselator reaction-diffusion system arising in chemical reactions, Math. Meth. Appl. Sci. 43 (2020) 3903. https://doi.org/10. $1002 / \mathrm{mma}$.

23. A. Bekir, and Ö. Güner, Cevikel A.C. Fractional Complex Transform and exp-Function Methods for Fractional Differential Equations, Abstr. Appl. Anal., 2013 (2013) 426462. https://doi.org/10.1155/2013/426462.

24. Ö. Güner and A. C. Cevikel, A Procedure to Construct Exact Solutions of Nonlinear Fractional Differential Equations, Sci. World J. 2014 (2014) 489495. https : / / doi . org/10 . $1155 / 2014 / 489495$.

25. A.C. Cevikel, New Exact Solutions of The Space-Time Fractional KdV-Burgers and Non-Linear Fractional Foam Drainage Equation, Therm. Sci., 22 (2018) 15. https://doi.org/ 10.2298/TSCI170615267C.

26. A. Bekir, Ö. Güner, and A. Cevikel, The Exp-function Method for Some Time-fractional Differential Equations, IEEE-CAA J. Autom. Sinica, 4 (2017) 315. https://doi.org/10. $1109 /$ JAS.2016.7510172.

27. A. Bekir, Ö. Güner, B. Ayhan, and A. C. Cevikel, Exact Solutions for Fractional Differential-Difference Equations by (G '/G)-Expansion Method with Modified Riemann-Liouville Derivative, Adv. Appl. Math. Mech., 8 (2016) 293. https : //doi.org/10.4208/aamm.2014.m798.

28. Ö. Güner, E. Aksoy, A. Bekir, and A. C. Cevikel, Different methods for (3+1)-dimensional space-time fractional modified KdV-Zakharov-Kuznetsov equation, Comput. Math. Appl., 71 (2016) 1259. https://doi.org/10.1016/j.camwa. 2016.02 .004 .

29. N. Shang, and B. Zheng, Exact Solutions for Three Fractional Partial Differential Equations by the $\left(G^{\prime} / G\right)$ Method, Int. J. Appl. Math. 43 (2013) 3.

30. Ö. Güner, A. Bekir, and A.C.Cevikel, A variety of exact solutions for the time fractional Cahn-Allen equation, Eur. Phys. J. Plus, 130 (2015) 7.https: //doi.org/10.1140/epjp/ i2015-15146-9

31. M. Eslami, B. F. Vajargah, M. Mirzazadeh, and A. Biswas, Application of first integral method to fractional partial differential equations, Indian J. Phys. 88 (2014) 177. https: //doi.org/10.1007/s12648-013-0401-6.
32. M. Eslami, and H. Rezazadeh, The first integral method for $\mathrm{Wu}$-Zhang system with conformable time-fractional derivative. Calcolo, 53 (2016) 475. https://doi.org/10.1007/ s10092-015-0158-8.

33. E. Akso, A.C. Cevikel, A. Bekir, Soliton solutions of $(2+1)-$ dimensional time-fractional Zoomeron equation, Optik, 127 (2016) 6933. https://doi.org/10.1016/j.ijleo. 2016.04 .122 .

34. B. Zheng, C.Wen, B.Zheng, C.Wen, Exact solutions for fractional partial differential equations by a new fractional subequation method, Adv. Differ. Equ., 2013 (2013) 199. https: //doi.org/10.1186/1687-1847-2013-199.

35. J. F. Alzaidy, Fractional Sub-Equation Method and its Applications to the Space-Time Fractional Differential Equations in Mathematical Physics, Br. J. Math. Comput. Sci., 3 (2013) 153. https://doi.org/10.9734/BJMCS/2013/2908.

36. A. Bekir, E. Aksoy, A.C. Cevikel, Exact solutions of nonlinear time fractional partial differential equations by sub-equation method, Math. Meth. Appl. Sci. 38 (2015) 13. 2779. https: //doi.org/10.1002/mma.3260.

37. E. Aksoy, A. Bekir, A.C. Cevikel, Study on Fractional Differential Equations with Modified Riemann-Liouville Derivative via Kudryashov Method, Int. J. Nonlinear Sci. Numer. Simul., 20 (2019) 511. https://doi.or10.1515/ ijnsns-2015-0151.

38. A. Zerarka, S. Ouamane, and A. Attaf, On the functional variable method for finding exact solutions to a class of wave equations, Appl. Math. Comput., 217 (2010) 289. https: //doi.org/10.1016/j.amc.2010.08.070.

39. N. Taghizadeh, M. Mirzazadeh, M. Rahimian, and M. Akbari Application of the simplest equation method to some timefractional partial differential equations, Ain Shams Eng. J., 4 (2013) 897. https://doi.org/10.1016/j.asej. 2013.01 .006 .

40. A. Esen, N. M. Yagmurlu, O.Tasbozan, Approximate Analytical Solution to Time-Fractional Damped Burger and CahnAllen Equations, Appl. Math. Inf. Sci. 7 (2013) 1951. https : //doi.org/10.12785/amis/070533.

41. G. Jumarie, Modified Riemann-Liouville derivative and fractional Taylor series of nondifferentiable functions further results, Comput. Math. Appl., 51 (2006) 1367. https://doi. org/10.1016/j.camwa.2006.02.001.

42. G. Jumarie, Table of some basic fractional calculus formulae derived from a modified Riemann-Liouvillie derivative for nondifferentiable functions, Appl. Maths. Lett. 22 (2009) 378. https://doi.org/10.1016/j.aml.2008.06. 003.

43. H. Bulut and B. Kilinç Exact solutions for some fractional nonlinear partial differential equations via Kudryashov mothod, $J$. New World Sci. Acad., 8 (2013) 24. http: //dx.doi.org/ 10.0000/NWSA.2013.8.1.3A0063.

44. Z.B. Li and J H. He, Fractional complex transform for fractional differential equations, Math. Comput. Appl., 15 (2010) 970. https://doi.org/10.3390/mca15050970.

45. Z.B. Li, and J. H. He, Application of the fractional complex transform to fractional differential equations Nonlinear Sci. Lett. A Math. Phys. Mech., 2 (2011) 121. https://doi. org/10.1186/1687-1847-2013-97. 
46. N.A. Kudryashov, One method for finding exact solutions of nonlinear differential equations, Commun. Nonlinear Sci.
Numer. Simul. 17 (2012) 2248. https://doi.org/10. $1016 / j . c n s n s .2011 .10 .016$. 